\title{
Singularly perturbed convection-diffusion boundary value problems with two small parameters using nonpolynomial spline technique
}

\author{
Pooja Khandelwal $^{1} \cdot$ Arshad Khan $^{1}$
}

Received: 21 August 2016/Accepted: 31 January 2017/Published online: 4 March 2017

(c) The Author(s) 2017. This article is published with open access at Springerlink.com

\begin{abstract}
In this paper, a new nonpolynomial cubic spline method is developed for solving two-parameter singularly perturbed boundary value problems. Convergence analysis is briefly discussed. Numerical examples and computational results illustrate and guarantee a higher accuracy by this technique. Comparisons are made to confirm the reliability and accuracy of the proposed technique.
\end{abstract}

Keywords Singular perturbation - Nonpolynomial cubic spline - Convergence analysis · Boundary value problem . Convection-diffusion

\section{Introduction}

We consider the two-parameter singularly perturbed convection-diffusion boundary value problems of the form:

$L y(x) \equiv-\epsilon y^{\prime \prime}(x)+\mu p(x) y^{\prime}(x)+f(x) y(x)=g(x), \quad x \in(a, b)$

subjected to the boundary conditions:

$y(0)=\alpha_{0}, \quad y(1)=\alpha_{1}$.

with two small positive parameters $0<\epsilon \ll 1,0<\mu \ll 1$, where $p(x), f(x)$, and $g(x)$ are sufficiently smooth real

Pooja Khandelwal

pooja2n@gmail.com

Arshad Khan

akhan1234in@rediffmail.com

1 Department of Mathematics, Jamia Millia Islamia, New Delhi 110025, India valued functions with $p(x) \geq p^{*}>0, f(x) \geq f^{*}>0$, and $g(x) \geq g^{*}>0$ for $x \in(a, b)$. Under these assumptions, problem (1.1) is characterized into two cases:

1. For $\mu=0$, problem (1.1) becomes reaction-diffusion problem.

2. For $\mu=1$, problem (1.1) becomes convection-diffusion problem.

This type of problem arises in the fields like engineering, mathematical physics, and in many areas of applied mathematics. We often come across boundary value problems in which one or small positive parameter multiplies with the derivatives. A large number of research papers have been found in the literature for single parameter convection-diffusion and reaction-diffusion problems [2, 8, 9, 12, 16]. However, only a very few authors have discussed two-parameter singularly perturbed boundary value problems $[4,6,7$, $10,11,14,16,18-20]$. The nature of two parameters is asymptotically examined by O' Malley [14]. Different numerical methods have been proposed by various authors for two-parameter singularly perturbed problems such as exponentially fitted cubic spline method [7], finite difference, finite element, and B-spline collocation method [6, 11], Haar wavelet method [16], and exponential spline technique [18]. For more information about SPPs, readers are referred to books [13, 15] and references therein.

In this paper, we introduce a new nonpolynomial cubic spline method as an alternative to existing methods. The paper is organised into five sections. In Sect. 2, we give a brief derivation of nonpolynomial parameters cubic spline. In Sect. 3, we presented the formulation of the method. Convergence analysis is briefly discussed in Sect. 4. Finally, in Sect. 5, numerical examples and comparison 
with the existing methods are given that demonstrate the practical applicability and superiority of the proposed method.

\section{Nonpolynomial spline function}

We consider a uniform mesh $\Delta$ with nodal points $x_{i}$ on $[a, b]$, such that $\Delta: a=x_{0}<x_{1}<x_{2}<, \cdots,<x_{n}-1<$ $x_{n}=b$, where $x_{i}=a+i h, i=0,1, \ldots, n$, and $h=\frac{(b-a)}{n}$. A nonpolynomial spline function $S_{\Delta}(x)$ of class $C^{2}[a, b]$ which interpolates $y(x)$ at mesh points $x_{i}, i=0(1) n$ depends on a parameter $k$, if we take $k \rightarrow 0$, then it reduces to ordinary cubic spline in $[a, b]$.

For each segment $\left[x_{i}, x_{i+1}\right], i=0,1,2 \ldots n-1$, we consider the nonpolynomial cubic spline $S_{\triangle}(x)$ of the form:

$$
\begin{aligned}
S_{\triangle}(x)= & a_{i} \sin k\left(x-x_{i}\right)+b_{i} \cos k\left(x-x_{i}\right) \\
& +c_{i} \mathrm{e}^{k\left(x-x_{i}\right)}+d_{i} \mathrm{e}^{-k\left(x-x_{i}\right)}, \quad i=0,1, \ldots, n,
\end{aligned}
$$

where $a_{i}, b_{i}, c_{i}$, and $d_{i}$ are unknown coefficients and $k$ is a free parameter which will be used to raise the accuracy of the method.

Let $y(x)$ be the exact solution and $y_{i}$ be an approximation to $y\left(x_{i}\right)$, obtained by the segment $S_{i}(x)$ of the mixed splines function passing through the points $\left(x_{i}, y_{i}\right)$ and $\left(x_{i+1}, y_{i+1}\right)$. To determine the coefficients of Eq. (2.1) in terms of $y_{i}, y_{i+1}, M_{i}, M_{i+1}$, we first define:

$$
\left.\begin{array}{rl}
S_{\triangle}\left(x_{i}\right)=y_{i}, & S_{\triangle}\left(x_{i+1}\right)=y_{i+1}, \\
S_{\triangle}^{\prime \prime}\left(x_{i}\right)=M_{i}, & S_{\triangle}^{\prime \prime}\left(x_{i+1}\right)=M_{i+1} .
\end{array}\right\}
$$

We obtain via a long but straightforward calculation

$$
\begin{aligned}
a_{i}= & \frac{\left(k^{2} y_{i+1}-M_{i+1}\right)-\cos \theta\left(k^{2} y_{i}-M_{i}\right)}{2 k^{2} \sin \theta}, \\
b_{i}= & \frac{\left(k^{2} y_{i}-M_{i}\right)}{2 k^{2}}, \\
c_{i}= & \frac{\mathrm{e}^{\theta}\left(k^{2} y_{i+1}+M_{i+1}\right)-\left(k^{2} y_{i}+M_{i}\right)}{2 k^{2}\left(\mathrm{e}^{2 \theta}-1\right)}, \\
d_{i}= & \frac{\mathrm{e}^{2 \theta}\left(k^{2} y_{i}+M_{i}\right)-\mathrm{e}^{\theta}\left(k^{2} y_{i+1}+M_{i+1}\right)}{2 k^{2}\left(\mathrm{e}^{2 \theta}-1\right)}, \\
& \theta=k h \quad \text { and } \quad i=0(1) n-1 .
\end{aligned}
$$

Using the continuity of the first derivative at the point $x=x_{i}$, we obtain the following tridiagonal system for $i=1,2, \ldots, n-1$ :

$$
y_{i-1}+\gamma y_{i}+y_{i+1}=h^{2}\left(\alpha M_{i-1}+\beta M_{i}+\alpha M_{i+1}\right),
$$

where

$$
\begin{aligned}
& \alpha=\frac{\left(\mathrm{e}^{2 \theta}-2 \mathrm{e}^{\theta} \sin \theta-1\right)}{\theta^{2}\left(\mathrm{e}^{2 \theta}+2 \mathrm{e}^{\theta} \sin \theta-1\right)}, \\
& \beta=2 \frac{\left[\mathrm{e}^{2 \theta}(\sin \theta-\cos \theta)-(\sin \theta+\cos \theta)\right]}{\theta^{2}\left(\mathrm{e}^{2 \theta}+2 \mathrm{e}^{\theta} \sin \theta-1\right)}, \\
& \gamma=-2 \frac{\left[\mathrm{e}^{2 \theta}(\sin \theta+\cos \theta)+(\sin \theta-\cos \theta)\right]}{\theta^{2}\left(\mathrm{e}^{2 \theta}+2 \mathrm{e}^{\theta} \sin \theta-1\right)} .
\end{aligned}
$$

If $\theta \rightarrow 0$, then $(\alpha, \beta, \gamma) \rightarrow\left(\frac{1}{6}, \frac{4}{6},-2\right)$, and then spline defined by (2.3) reduces to a ordinary cubic spline relation [13]:

$$
\left(y_{i-1}-2 y_{i}+y_{i+1}\right)=\frac{h^{2}}{6}\left(M_{i-1}+4 M_{i}+M_{i+1}\right) \text {. }
$$

The relation (2.3) gives $(n-1)$ linear algebraic equations in $(n-1)$ unknowns $y_{i}, i=1,2, \ldots, n-1$.

\section{The method}

At the grid point $x_{i}$, the proposed two-parameter singularly perturbed boundary value problem (1.1) can be discretized as follows:

$-\epsilon y^{\prime \prime}\left(x_{i}\right)+\mu p\left(x_{i}\right) y^{\prime}\left(x_{i}\right)+f\left(x_{i}\right) y\left(x_{i}\right)=g\left(x_{i}\right)$.

Using spline's second derivative, we have

$$
\begin{aligned}
& M_{i}=\frac{\mu p_{i} y_{i}^{\prime}+f_{i} y_{i}-g_{i}}{\epsilon}, \\
& M_{i-1}=\frac{\mu p_{i-1} y_{i-1}^{\prime}+f_{i-1} y_{i-1}-g_{i-1}}{\epsilon}, \\
& M_{i+1}=\frac{\mu p_{i+1} y_{i+1}^{\prime}+f_{i+1} y_{i+1}-g_{i+1}}{\epsilon},
\end{aligned}
$$

where

$$
\begin{aligned}
& y_{i}^{\prime}=\frac{y_{i+1}-y_{i-1}}{2 h}, \\
& y_{i-1}^{\prime}=\frac{-y_{i+1}+4 y_{i}-3 y_{i-1}}{2 h}, \quad y_{i+1}^{\prime}=\frac{3 y_{i+1}-4 y_{i}+y_{i-1}}{2 h},
\end{aligned}
$$

$p_{i}=p\left(x_{i}\right), f_{i}=f\left(x_{i}\right)$ and $g_{i}=g\left(x_{i}\right)$.

Substituting the values of $M_{j}(j=i, i \pm 1)$ in Eq. (2.3), we have

$$
\begin{aligned}
& {\left[-\epsilon+\frac{\mu h}{2}\left(-3 \alpha p_{i-1}-\beta p_{i}+\alpha p_{i+1}\right)+h^{2} \alpha f_{i-1}\right] y_{i-1}} \\
& \quad+\left[-\gamma \epsilon+\frac{\mu h}{2}\left(4 \alpha p_{i-1}-4 \alpha p_{i+1}\right)+h^{2} \beta f_{i}\right] y_{i} \\
& \quad+\left[-\epsilon+\frac{\mu h}{2}\left(-\alpha p_{i-1}+\beta p_{i}+3 \alpha p_{i+1}\right)+h^{2} \alpha f_{i+1}\right] y_{i+1} \\
& \quad=h^{2}\left(\alpha g_{i-1}+\beta g_{i}+\alpha g_{i+1}\right), \quad i=1(1) n-1 .
\end{aligned}
$$


Finally, we arrive at the following system:

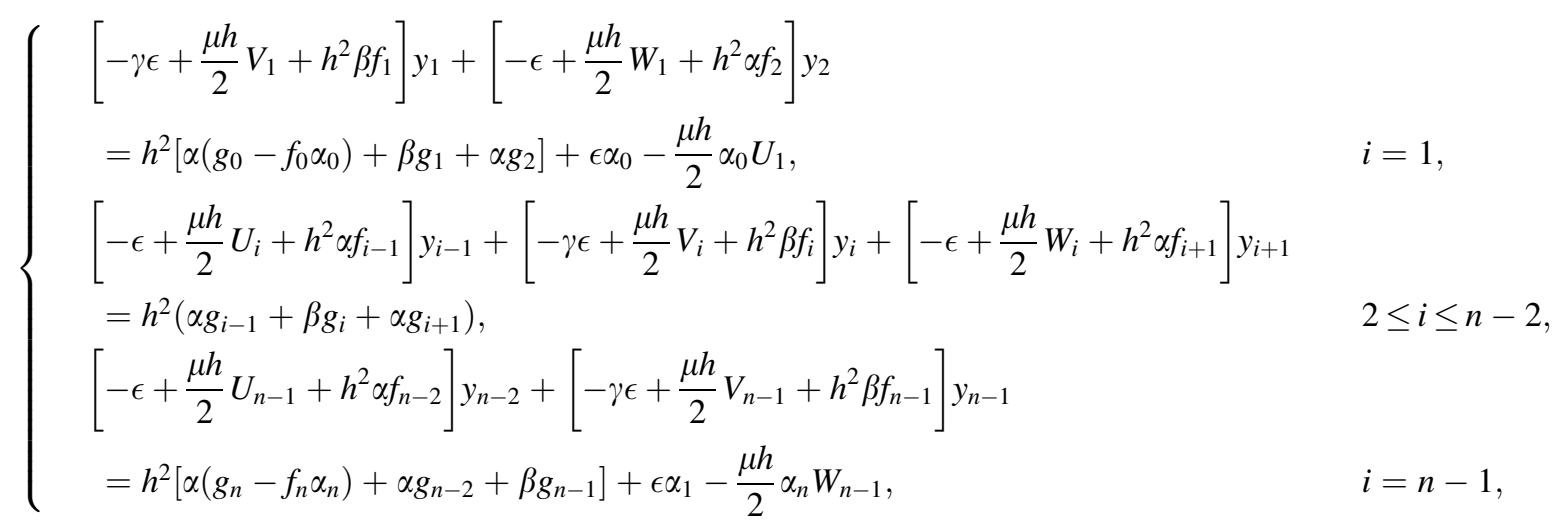

where

$$
\begin{gathered}
U_{i}=\left(-3 \alpha p_{i-1}-\beta p_{i}+\alpha p_{i+1}\right), \quad V_{i}=\left(4 \alpha p_{i-1}-4 \alpha p_{i+1}\right) \\
W_{i}=\left(-\alpha p_{i-1}+\beta p_{i}+3 \alpha p_{i+1}\right), \quad i=1(1) n-1
\end{gathered}
$$

\section{Convergence analysis}

In this section, we investigate the convergence analysis of the proposed method. For this, let $Y=y\left(x_{i}\right), \bar{Y}=\left(y_{i}\right)$, $C=\left(c_{i}\right), T=\left(t_{i}\right), E=\left(e_{i}\right)=Y-\bar{Y}, i=1,2, \ldots, n-1$ be an exact column vectors, where $Y, \bar{Y}, T$, and $E$ are exact, approximate, local truncation error, and discretization error, respectively.

We can write the standard matrix equation for the method developed in the following form:

$$
M \bar{Y}=C,
$$

where $M$ is a matrix of order $(n-1)$ with

$$
M=\left(A_{0}+A_{1}+h^{2} A_{2} F\right) \text {. }
$$

The tridiagonal matrices $A_{0}, A_{1}$, and $A_{2}$ have the form:

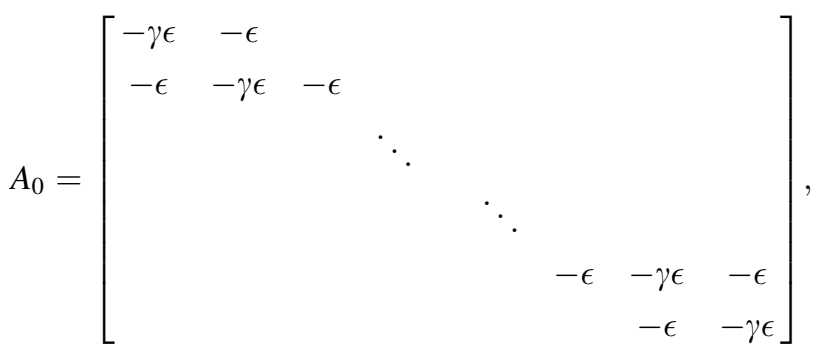

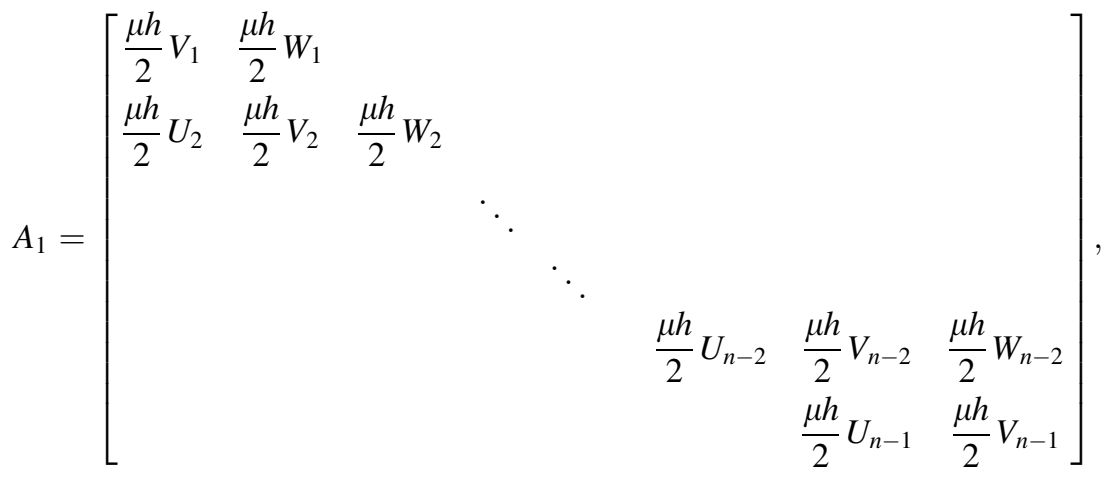


$A_{2}=\left[\begin{array}{cccccccc}\beta & \alpha & & & & & \\ \alpha & \beta & \alpha & & & & \\ & & & \ddots & & & & \\ & & & & \ddots & & & \\ & & & & \alpha & \beta & \alpha \\ & & & & & \alpha & \beta\end{array}\right]$,

and

$$
F=\left[\begin{array}{c}
f_{1} \\
f_{2} \\
\vdots \\
\vdots \\
f_{n-2} \\
f_{n-1}
\end{array}\right]
$$

For the $(n-1)$ column vector $C$, we have
From Eqs. (4.1) and (4.8), we get

$$
M(Y-\bar{Y})=T(h)
$$

or

$M E=T(h)$,

where $E=(Y-\bar{Y})=\left[e_{1}, e_{2}, \ldots, e_{n-1}\right]^{T}$.

Clearly, the row sums $M_{1}, M_{2}, \ldots, M_{n-1}$ of $M$ are

$$
\begin{gathered}
M_{1}=-\gamma \epsilon-\epsilon+\frac{\mu h}{2}\left(3 \alpha p_{0}+\beta p_{1}-\alpha p_{2}\right)+h^{2}\left(\beta f_{1}+\alpha f_{2}\right), \quad i=1, \\
M_{i}=-\gamma \epsilon-2 \epsilon+h^{2}\left(\alpha f_{i-1}+\beta f_{i}+\alpha f_{i+1}\right), \quad i=2(1) n-2, \\
M_{n-1}=-\gamma \epsilon-\epsilon+\frac{\mu h}{2}\left(\alpha p_{n-2}-\beta p_{n-1}-3 \alpha p_{n}\right) \\
\quad+h^{2}\left(\alpha f_{n-2}+\beta f_{n-1}\right), \quad i=n-1,
\end{gathered}
$$

If we choose $h$ sufficiently small, matrix $M$ becomes irreducible and monotone [5]. It follows that $M^{-1}$ exists

$c_{i}= \begin{cases}h^{2}\left[\alpha\left(g_{0}-f_{0} \alpha_{0}\right)+\beta g_{1}+\alpha g_{2}\right]+\epsilon \alpha_{0}-\frac{\mu h}{2} \alpha_{0} U_{1}, & i=1, \\ h^{2}\left(\alpha g_{i-1}+\beta g_{i}+\alpha g_{i+1}\right), & 2 \leq i \leq n-2, \\ h^{2}\left[\alpha\left(g_{n}-f_{n} \alpha_{n}\right)+\alpha g_{n-2}+\beta g_{n-1}\right]+\epsilon \alpha_{1}-\frac{\mu h}{2} \alpha_{n} W_{n-1}, & i=n-1 .\end{cases}$

Now, considering the above system with exact solution $Y=\left[y\left(x_{1}\right), y\left(x_{2}\right), \ldots, y\left(x_{n-1}\right)\right]$, we have

$$
M Y=T(h)+C,
$$

where $T(h)=\left[t_{1}(h), t_{2}(h), \ldots, t_{n-1}(h)\right]^{T}$ is the local truncation error vector, where

$$
\begin{gathered}
t_{i}(h)=-(2+\gamma) \epsilon y_{i}+(2 \alpha+\beta-1) \epsilon h^{2} y^{(2)}\left(\xi_{i}\right)+\left(\alpha-\frac{1}{12}\right) \\
\epsilon h^{4} y^{(4)}\left(\xi_{i}\right)+\left(\frac{\alpha}{12}-\frac{1}{360}\right) \epsilon h^{6} y^{(6)}\left(\xi_{i}\right), \quad x_{i-1}<\xi_{i}<x_{i+1} .
\end{gathered}
$$

for any arbitrary choice of $\alpha, \beta$, and $\gamma$ except $\alpha=1 / 6, \beta=$ $4 / 6$ and $\gamma=-2$.

If we choose $\alpha=1 / 6, \beta=4 / 6$, and $\gamma=-2$,

$t_{i}(h)=\frac{\epsilon h^{4}}{12} y^{(4)}\left(\xi_{i}\right), \quad x_{i-1}<\xi_{i}<x_{i+1}$.

If we choose $\alpha=1 / 12, \beta=10 / 12$, and $\gamma=-2$,

$t_{i}(h)=\frac{\epsilon h^{6}}{240} y^{(6)}\left(\xi_{i}\right), \quad x_{i-1}<\xi_{i}<x_{i+1}$. and its elements are nonnegative. Hence, from Eq. (4.12), we have

$E=M^{-1} T(h)$

Let $m_{k, i}^{-1}$ is the $(k, i)^{\text {th }}$ element of the matrix $M^{-1}$. We define

$\left\|m_{k, i}^{-1}\right\|=\max _{1 \leq k \leq n} \sum_{i=1}^{n-1}\left|m_{k, i}^{-1}\right|$

and

$\|T\|=\max _{1 \leq k \leq n}\left|t_{k}\right|$

In addition, from the theory of matrices, we have

$\sum_{i=1}^{n-1} m_{k, i}^{-1} M_{i}=1, \quad k=1,2, \ldots, n-1$.

Therefore

$m_{k, i}^{-1} \leq \frac{1}{\min _{1 \leq i \leq n-1} M_{i}}=\frac{1}{h^{2} Q_{i_{o}}}$,

where $Q_{i_{o}}=\frac{1}{h^{2}} \min _{i} M_{i}>0$, for some $i_{o}$ between 1 to $n-1$. 
From Eqs. (4.9), (4.13), and (4.14), we have

$e_{i}=\sum_{i=1}^{n-1} m_{k, i}^{-1} T_{i}(h), \quad k=1,2, \ldots, n-1$

and therefore

$\left|e_{i}\right| \leq \frac{K h^{2}}{\left|Q_{i_{o}}\right|}, \quad i=1,2, \ldots, n-1$,

where $K$ is a constant independent of $h$. It follows that $\|E\|=O\left(h^{2}\right)$.

However, for the choice of parameters, $\alpha=1 / 12, \beta=$ $10 / 12$, and $\gamma=-2$,

$\left|e_{i}\right| \leq \frac{K h^{4}}{\left|Q_{i_{o}}\right|}, \quad i=1,2, \ldots, n-1$,

where $K$ is a constant independent of $h$. It follows that $\|E\|=O\left(h^{4}\right)$.

We summarize the above result in the following theorem:

Theorem 4.1 Let $y(x)$ be the exact solution of two-parameter singularly perturbed boundary value problem (1.1) and let $y_{i}$ be the numerical solution obtained from the difference scheme (4.1). Then, for sufficiently small $h$, scheme gives a second-order convergent solution for any arbitrary choice of $\alpha$ and $\beta$ with $\gamma=-2$ and a fourth-order convergent solution for $\alpha=1 / 12, \beta=10 / 12$, and $\gamma=-2$.

\section{Numerical examples}

To test the viability of the proposed method based on nonpolynomial cubic spline, two numerical examples are considered. All the computations were performed using MATLAB. We also compare our method with the existing methods which shown improvement.

Example 1 Consider the following two-parameter singularly perturbed boundary value problem, which is discussed in $[12,19]$ :

$-\epsilon y^{\prime \prime}+\mu y^{\prime}+y=1, \quad x \in(0,1)$, subjected to the boundary conditions:

$y(0)=0, \quad y(1)=0$.

The exact solution of the above problem is

$y(x)=\frac{\left(\mathrm{e}^{\lambda_{2}}-1\right) \mathrm{e}^{\lambda_{1} x}}{\mathrm{e}^{\lambda_{1}}-\mathrm{e}^{\lambda_{2}}}+\frac{\left(1-\mathrm{e}^{\lambda_{1}}\right) \mathrm{e}^{\lambda_{2} x}}{\mathrm{e}^{\lambda_{1}}-\mathrm{e}^{\lambda_{2}}}+1$,

where

$\lambda_{1}=\frac{1+\sqrt{1+4 \epsilon}}{2 \epsilon}, \quad \lambda_{2}=\frac{1-\sqrt{1+4 \epsilon}}{2 \epsilon}$.

Example 2 Consider the following two-parameter singularly perturbed boundary value problem, which is discussed in $[6,16,19]$ :

$-\epsilon y^{\prime \prime}+\mu y^{\prime}+y=\cos (\pi x), \quad x \in(0,1)$,

subjected to the boundary conditions:

$y(0)=0, \quad y(1)=0$.

The exact solution of the above problem is

$$
u(x)=\rho_{1} \cos (\pi x)+\rho_{2} \sin (\pi x)+\psi_{1} \mathrm{e}^{\lambda_{1} x}+\psi_{2} \mathrm{e}^{-\lambda_{2}(1-x)},
$$

where

$$
\begin{aligned}
& \rho_{1}=\frac{\epsilon \pi^{2}+1}{\epsilon^{2} \pi^{2}+\left(\epsilon \pi^{2}+1\right)^{2}}, \quad \rho_{2}=\frac{\epsilon \pi}{\epsilon^{2} \pi^{2}+\left(\epsilon \pi^{2}+1\right)^{2}}, \\
& \psi_{1}=-\rho_{1} \frac{1+\mathrm{e}^{-\lambda_{2}}}{1-\mathrm{e}^{\lambda_{1}-\lambda_{2}}}, \quad \psi_{2}=\rho_{1} \frac{1+\mathrm{e}^{\lambda_{1}}}{1-\mathrm{e}^{\lambda_{1}-\lambda_{2}}}, \\
& \lambda_{1}=\frac{\mu-\sqrt{\mu^{2}+4 \epsilon}}{2 \epsilon}, \quad \lambda_{2}=\frac{\mu+\sqrt{\mu^{2}+4 \epsilon}}{2 \epsilon} .
\end{aligned}
$$

The numerical results corresponding to the Examples 1 and 2 are briefly summarized in Tables 1, 2, 3, and 4, and Figs. 1, 2, 3, and 4. Comparison with other existing methods are also listed in Tables 1, 2, 3 and 4. These tables show that method is more accurate than the existing methods.
Table 1 Comparison of pointwise errors, Example 1

\begin{tabular}{lllll}
\hline $\mathbf{x} \downarrow$ & $\begin{array}{l}\epsilon=0.1, \mu=1, \mathrm{n}=32 \\
\text { Lin et al. [12] }\end{array}$ & Our method & $\begin{array}{l}\epsilon=0.1, \mu=1, \mathrm{n}=128 \\
\text { Lin et al. [12] }\end{array}$ & Our method \\
\hline $1 / 16$ & $2.74(-2)$ & $3.27(-6)$ & $6.8(-3)$ & $1.15(-6)$ \\
$2 / 16$ & $2.59(-2)$ & $5.44(-6)$ & $6.4(-3)$ & $2.18(-6)$ \\
$4 / 16$ & $2.30(-2)$ & $3.98(-6)$ & $5.7(-3)$ & $3.72(-6)$ \\
$6 / 16$ & $2.04(-2)$ & $1.61(-5)$ & $5.0(-3)$ & $3.92(-6)$ \\
$12 / 16$ & $2.50(-2)$ & $8.52(-4)$ & $4.0(-4)$ & $4.45(-5)$ \\
$14 / 16$ & $3.30(-2)$ & $1.70(-3)$ & $9.4(-3)$ & $9.61(-5)$ \\
\hline
\end{tabular}


Table 2 Comparison of pointwise errors, Example 1

\begin{tabular}{lllll}
\hline $\mathbf{x} \downarrow$ & $\begin{array}{l}\epsilon=0.01, \mu=1, \mathrm{n}=32 \\
\text { Lin et al. [12] }\end{array}$ & Our method & $\begin{array}{l}\epsilon=0.01, \mu=1, \mathrm{n}=128 \\
\text { Lin et al. [12] }\end{array}$ & Our method \\
\hline $1 / 16$ & $2.95(-2)$ & $4.55(-6)$ & $7.3(-3)$ & $2.84(-7)$ \\
$2 / 16$ & $2.78(-2)$ & $8.55(-6)$ & $6.9(-3)$ & $5.35(-7)$ \\
$4 / 16$ & $2.45(-2)$ & $1.51(-5)$ & $6.1(-3)$ & $9.45(-7)$ \\
$6 / 16$ & $2.17(-2)$ & $2.00(-5)$ & $5.4(-3)$ & $1.25(-6)$ \\
$12 / 16$ & $1.50(-2)$ & $3.07(-5)$ & $3.7(-3)$ & $1.73(-6)$ \\
$14 / 16$ & $1.29(-2)$ & $1.41(-3)$ & $3.3(-3)$ & $7.31(-7)$ \\
\hline
\end{tabular}

Table 3 Comparison of maximum absolute errors, Example 2

\begin{tabular}{lllll}
\hline $\boldsymbol{\mu} \downarrow$ & $\begin{array}{l}\epsilon=10^{-2}, \mathrm{n}=128 \\
\text { Kadalbajoo et al. [6] }\end{array}$ & Zahra et al. [19] & Pandit et al. [16] & Our method \\
\hline $10^{-3}$ & $8.3832(-5)$ & $4.1924(-5)$ & $4.2303(-5)$ & $6.0243(-6)$ \\
$10^{-4}$ & $8.2686(-5)$ & $4.1296(-5)$ & $4.1318(-5)$ & $6.1827(-7)$ \\
$10^{-5}$ & $8.2572(-5)$ & $4.1232(-5)$ & $4.1220(-5)$ & $1.1455(-7)$ \\
$10^{-6}$ & $8.2561(-5)$ & $4.1226(-5)$ & $4.1210(-5)$ & $7.2269(-8)$ \\
$10^{-7}$ & $8.25596(-5)$ & $4.1225(-5)$ & $4.1209(-5)$ & $6.8266(-8)$ \\
\hline
\end{tabular}

Table 4 Comparison of maximum absolute errors, Example 2

\begin{tabular}{llllr}
\hline $\boldsymbol{\mu} \downarrow$ & $\begin{array}{l}\epsilon=10^{-4}, \mathrm{n}=128 \\
\text { Kadalbajoo et al. [6] }\end{array}$ & Zahra et al. [19] & Pandit et al. [16] & Our method \\
\hline $10^{-3}$ & $9.4446(-3)$ & $4.7598(-3)$ & $5.1964(-3)$ & $6.2154(-3)$ \\
$10^{-4}$ & $9.0436(-3)$ & $4.2856(-3)$ & $4.1710(-3)$ & $1.8330(-3)$ \\
$10^{-5}$ & $9.0036(-3)$ & $4.2295(-3)$ & $4.0754(-3)$ & $1.1412(-3)$ \\
$10^{-6}$ & $8.9996(-3)$ & $4.2238(-3)$ & $4.0659(-3)$ & $1.3699(-3)$ \\
$10^{-7}$ & $8.9992(-3)$ & $4.2232(-3)$ & $4.0650(-3)$ & $1.3656(-3)$ \\
\hline
\end{tabular}

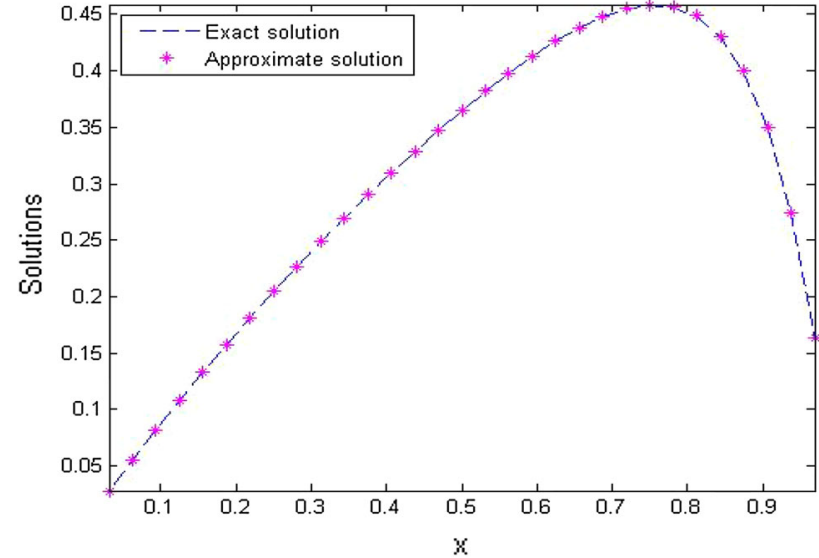

Fig. 1 Physical behaviour of numerical solution of Example 1 for $\epsilon=0.1, \mu=1$, and $n=32$

Tables 1, 2 show the pointwise errors at different values of $n$ and for small values of $\epsilon$. Tables 3, 4 show the maximum absolute errors of the Example 2 for different values of $\epsilon$ and $\mu$. Figures 1,2 compare the exact and approximate

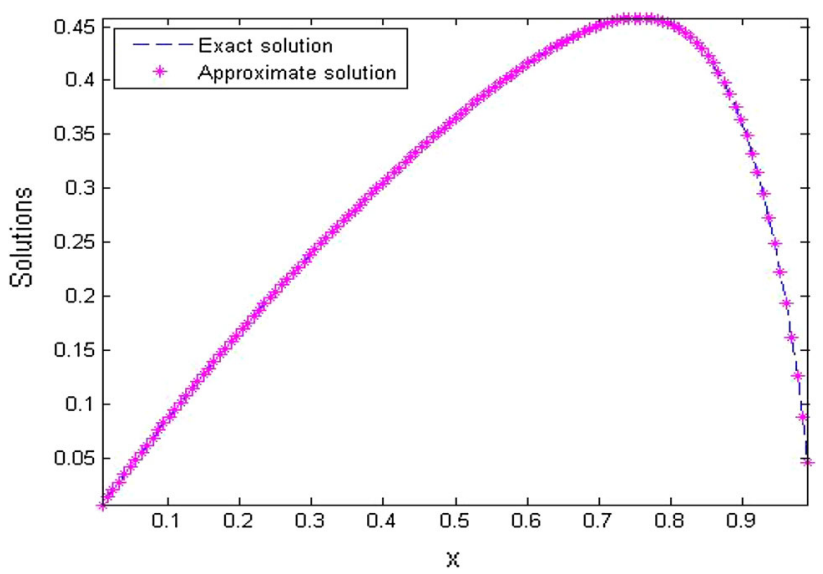

Fig. 2 Physical behaviour of numerical solution of Example 1 for $\epsilon=0.1, \mu=1$, and $n=128$

solutions of Example 1 for $\epsilon=0.1$ and $\mu=1$, while Figs. 3 and 4 report the exact and approximate solutions of Example 2 for different values of $\epsilon$ and $\mu$. 


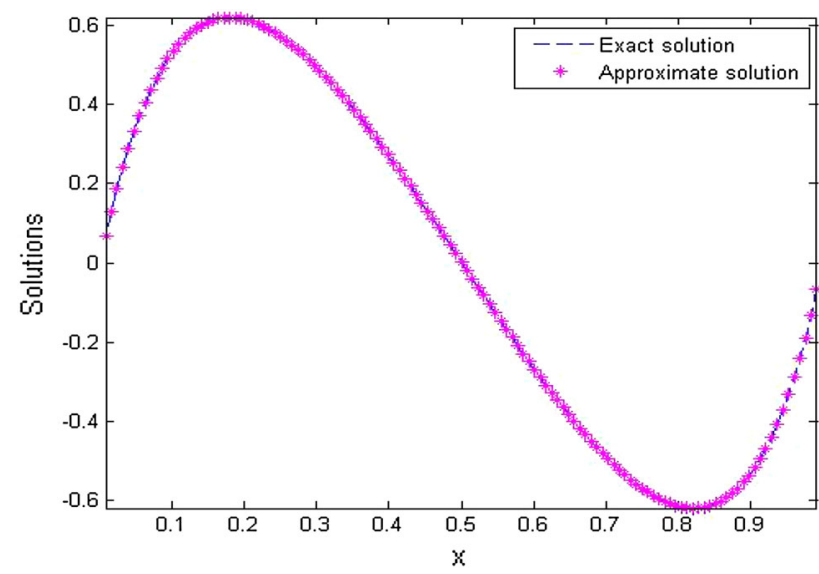

Fig. 3 Physical behaviour of numerical solution of Example 2 for $\epsilon=10^{-2}, \mu=10^{-3}$, and $n=128$

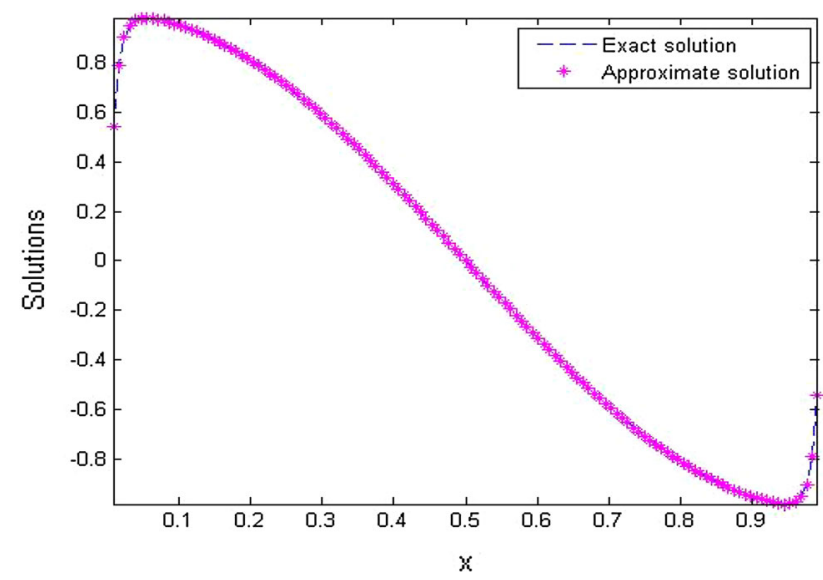

Fig. 4 Physical behaviour of numerical solution of Example 2 for $\epsilon=10^{-4}, \mu=10^{-5}$, and $n=128$

\section{Concluding remarks}

In this paper, nonpolynomial cubic spline function is used for finding the numerical solution of two-parameter convection-diffusion singularly perturbed boundary value problems. The computations associated with the examples discussed above were performed using MATLAB. The proposed method is computationally efficient and the algorithm can be easily implemented on a computer. Comparison of the method is also depicted through Tables 1, 2, 3, and 4 which shown that our methods perform better in the sense of accuracy and applicability. The solution profiles for the considered examples for different values of $\epsilon$ and $\mu$ are given in Figs. 1, 2, 3, and 4.

Acknowledgements The authors are thankful to referee for their valuable suggestions which improved the quality of the paper. The first author is also thankful to the NBHM, Department of Atomic Energy, Government of India, for its financial assistance vide letter no. $2 / 40(32) / 2012 / R \& D-I I / 11626$ to carry out this research work.
Open Access This article is distributed under the terms of the Creative Commons Attribution 4.0 International License (http://crea tivecommons.org/licenses/by/4.0/), which permits unrestricted use, distribution, and reproduction in any medium, provided you give appropriate credit to the original author(s) and the source, provide a link to the Creative Commons license, and indicate if changes were made.

\section{References}

1. Ahlberg, J.H., Nilson, E.N., Walsh, J.L.: The Theory of Splines and Their Applications. Academic Press, New York (1967)

2. Aziz, T., Khan, A.: A spline method for second-order singularly perturbed boundary-value problems. J. Comput. Appl. Math. 147, 445-452 (2002)

3. De Boor, C.: A practical guide to splines. In: Mathematics of computation, vol. 27(149). Springer-verlag (1978). doi:10.2307/ 2006241

4. Gracia, J.L., O'Riordan, E., Pickett, M.L.: A parameter robust second order numerical method for a singularly perturbed twoparameter problem. Appl. Numer. Math. 56, 962-980 (2006)

5. Henrici, P.: Discrete Variable Methods in Ordinary Differential Equations. Wiley, New York (1962)

6. Kadalbajoo, M.K., Yadaw, A.S.: B-spline collocation method for a two-parameter singularly perturbed convection-diffusion boundary value problems. Appl. Math. Comput. 201, 504-513 (2008)

7. Kadalbajoo, M.K., Jha, A.: Exponentially fitted cubic spline for two-parameter singularly perturbed boundary value problems. Int. J. Comput. Math. 89(6), 836-850 (2012)

8. Khan, A., Khan, I., Aziz, T.: Sextic spline solution of singularly perturbed boundary value problem. Appl. Math. Comput. 181, 432-439 (2006)

9. Khan, A., Khandelwal, P.: Non-polynomial sextic spline solution of singularly perturbed boundary-value problems. Int. J. Comput. Math. 19(5), 1122-1135 (2014)

10. Kumar, D., Yadaw, A.S., Kadalbajoo, M.K.: A parameter-uniform method for two parameter singularly perturbed boundary value problems via asymptotic expansion. Appl. Math. Inf. Sci. 7(4), 1525-1532 (2013)

11. LinB, T., Roos, H.G.: Analysis of a finite-difference scheme for a singularly perturbed problem with two small parameters. J. Math. Anal. Appl. 289, 355-366 (2004)

12. Lin, B., Li, K., Cheng, Z.: B-spline solution of a singularly perturbed boundary value problem arising in biology. Chaos Solitons Fractals 42, 2934-2948 (2009)

13. Miller, J.J.H., O'Riordan, E., Shishkin, G.I.: Fitted Numerical Methods for Singular Perturbation Problem. World Scientific, Singapore (1996)

14. O'Malley Jr., R.E.: Two parameter singular perturbation problems for second order equations. J. Math. Mech. 16, 1143-1164 (1967)

15. O'Malley Jr., R.E.: Introduction to Singular Perturbations. Academic Press, New York (1974)

16. Pandit, S., Kumar, M.: Haar wavelet approach for numerical solution of two parameters singularly perturbed boundary value problems. Appl. Math. Inf. Sci. 8(6), 2965-2974 (2014)

17. Rashidinia, J., Ghasemi, M., Mahmoodi, Z.: Spline approach to the solution of a singularly perturbed boundary value problems. Appl. Math. Comput. 189, 72-78 (2007)

18. Valarmathi, S., Ramanujam, N.: Computational methods for solving two-parameter singularly perturbed boundary value problems for second-order ordinary differential equations. Appl. Math. Comput. 136, 415-441 (2003) 
19. Zahra, W.K., El Mhlawy, A.M.: Numerical solution of two-parameter singularly perturbed boundary value problems via exponential spline. J. King Saud Univ. 25, 201-208 (2013)
20. Brdar, M., Zarin, H.: On a graded meshes for a two-parameter singularly perturbed problem. Appl. Math. Comput. 282, 97-107 (2016) 